\title{
Control of Simultaneous Osteogenic and Adipogenic Differentiation of Mesenchymal Stem Cells
}

\author{
Rong Cai ${ }^{1,2}$, Tomoko Nakamoto ${ }^{1}$, Takashi Hoshiba ${ }^{3}$, Naoki Kawazoe ${ }^{1}$ and Guoping Chen ${ }^{1,2 *}$ \\ ${ }^{1}$ Tissue Regeneration Materials Unit, International Center for Materials Nanoarchitectonics, National Institute for Materials Science, 1-1 Namiki, Tsukuba, Ibaraki 305- \\ 0044, Japan \\ ${ }^{2}$ Department of Materials Science and Engineering, Graduate School of Pure and Applied Sciences, Tsukuba University, 1-1-1 Tennodai, Tsukuba, Ibaraki 305-8577, \\ Japan \\ ${ }^{3}$ Department of Biochemical Engineering, Graduate School of Science and Engineering, Yamagata University, 4-3-16 Jonan, Yonezawa, Yamagata 992-8510, Japan
}

\begin{abstract}
Mesenchymal stem cells (MSCs) are pluripotent cells which can differentiate into several distinct lineages such as osteoblasts and adipocytes. Osteogenesis and adipogenesis of MSCs induced with specific induction factors have been well exploited. However, it is not clear how simultaneous osteogenesis and adipogenesis of MSCs is balanced by the composition of induction medium. In this study, MSCs were cultured in the mixture medium at different ratio of osteogenic medium (OM) and adipogenic medium (AM) to investigate the simultaneous osteogenesis and adipogenesis of MSCs. Differentiation was investigated by histological staining and real-time PCR analysis. The results showed that adipogenesis was dosage dependent on the concentration of adipogenic induction factors, while osteogenesis was not. The balance of osteogenesis and adipogenesis could be control by different ratio of osteogenic and adipogenic media. The results will be useful for controlling stem cell differentiation.
\end{abstract}

Keywords: Osteogenesis; Adipogenesis; Differentiation balance; Simultaneous differentiation; Mesenchymal stem cells

\section{Introduction}

Mesenchymal stem cells (MSCs) are pluripotent with the ability to differentiate into multiple cell lineages such as osteoblasts, adipocytes and chondrocytes [1]. MSCs have been used for tissue engineering and regenerative medicine. In vitro expansion and directed differentiation of MSCs are important for fundamental and clinical applications. The culture condition of isolation and in vitro expansion of MSCs have been extensively investigated [2-4]. It has been revealed that the composition of expansion medium, oxygen pressure, and culture substrate properties have significant effects on the biological properties of MSCs, such as proliferation, surface marker expression, and differentiation potential [5-7]. A combination of growth factors, extracellular matrix, physical stimuli, and culture conditions has been frequently used to investigate the differentiation of MSCs [8,9]. The soluble factors for MSCs differentiation are well established [1].

Adipogenic differentiation of MSCs occurs in medium containing dexamethasone (Dex), 3-isobutyl-1-methylxanthine (IBMX), insulin and indomethacin (Indo). To promote osteogenesis, Dex and $\beta$-glycerophosphate $(\beta$-GP) are usually supplemented in induction medium [9]. Although various factors have been used to investigate their effects on differentiation of MSCs, most of the studies focus on the MSCs differentiation into an individual lineage.

Osteogenesis and adipogenesis have been reported to have an inverse correlation [10]. Up-regulation of osteogenic differentiation is associated with down-regulation of adipognic differentiation and vice versa. Disruption of the balance between ostogenesis and adipogenesis may lead to some diseases, such as osteoporosis and diabetic bone loss [11-13]. Investigation of simultaneous osteogenesis and adipogenesis will provide some useful information for understanding the development process of the diseases related with osteogenesis and adipogenesis. It is not clear whether osteogenesis and adipogenesis of MSCs can be induced simultaneously and how the medium composition balances simultaneous osteogenesis and adipogenesis in the same medium. Therefore, in this study, MSCs were cultured in a mixture medium of osteogenic and adipogenic induction medium at various ratios and the balance of osteogenesis and adipogenesis of MSCs in this mixture medium was investigated.

\section{Materials and Methods}

\section{Preparation of mixture medium}

The osteogenic differentiation medium $(\mathrm{OM})$ was prepared by adding $10 \mathrm{nM}$ dexamethasone (Dex, Sigma, St. Louis, MO) and 10 $\mathrm{mM} \beta$-glycerophosphate ( $\beta$-GP, Sigma, St. Louis, MO) in Dulbecco's modified Eagle's medium (DMEM, Sigma, St. Louis, MO) with low glucose $(1000 \mathrm{mg} / \mathrm{L})$, containing $10 \%$ fetal bovine serum (FBS, Equitech-Bio, Kerrville, TX), glutamine $(584 \mathrm{mg} / \mathrm{L})$, penicillin $(100$ units $/ \mathrm{mL})$, streptomycin $(100 \mathrm{mg} / \mathrm{L})$, nonessential amino acids $(0.1$ $\mathrm{mM})$, sodium pryuvate $(1 \mathrm{mM})$, proline $(0.4 \mathrm{mM})$ and ascorbic acid $(50 \mathrm{mg} / \mathrm{L})$. The adipogenic differentiation medium (AM) was prepared by DMEM with high glucose $(4500 \mathrm{mg} / \mathrm{L})$ supplied with Dex $(1 \mu \mathrm{M})$, 3-isobutyl-1-methylxanthine (0.5 mM, IBMX), insulin $(10 \mu \mathrm{g} / \mathrm{mL})$ and indomethacin (100 mM, Indo). The mixture medium of osteogenic and adipogenic medium was prepared by combining $\mathrm{OM}$ and $\mathrm{AM}$ at different ratios (OM/AM (v/v)) of 100/0, 95/5, 90/10, 85/15, 80/20, $75 / 25,70 / 30,65 / 35,60 / 40,55 / 45,50 / 50,45 / 55,40 / 60,35 / 65,30 / 70$, $25 / 75,20 / 80,15 / 85,10 / 90,5 / 95$ and $0 / 100$. The concentration of induction factors of Dex, $\beta$-GP, IBMX, insulin and Indo was calculated from the mixture ratio (Figure 1).

*Corresponding author: Guoping Chen, Principal Investigator and Unit Director, Tissue Regeneration Materials Unit, International Center for Materials Nanoarchitectonics, National Institute for Materials Science, 1-1 Namiki, Tsukuba Ibaraki 3050044, Japan; Tel: +81-29-860-4496; Fax: +81-29-860-4714; E-mail: Guoping.Chen@nims.go.jp

Received July 14, 2014; Accepted August 01, 2014; Published August 03, 2014

Citation: Cai R, Nakamoto T, Hoshiba T, Kawazoe N, Chen G (2014) Control of Simultaneous Osteogenic and Adipogenic Differentiation of Mesenchymal Stem Cells. J Stem Cell Res Ther 4: 223. doi:10.4172/2157-7633.1000223

Copyright: (c) 2014 Cai R, et al. This is an open-access article distributed under the terms of the Creative Commons Attribution License, which permits unrestricted use, distribution, and reproduction in any medium, provided the original author and source are credited. 


\section{Simultaneous osteogenic and adipogenic differentiation of MSCs}

Human bone marrow-derived mesenchymal stem cells (MSCs, passage 2) were obtained from Lonza (Walkersville, MD) and subcultured twice in growth medium (Lonza, MD). The subcultured MSCs (at passage 4) were seeded in tissue culture plates (TCPS) at a density of 5,000 cells $/ \mathrm{cm}^{2}$ under an atmosphere of $5 \% \mathrm{CO}_{2}$ at $37^{\circ} \mathrm{C}$. The mixture medium was used and refreshed every 3 days during culture. The cells were cultured in mixture medium for 3, 7, 14, and 21 days.

\section{Alkaline phosphatase (ALP) staining}

After incubation in mixture medium for 1,2 and 3 weeks, the cells were washed with phosphate-buffered saline (PBS) and fixed with $4 \%$ paraformaldehyde in PBS for $10 \mathrm{~min}$. And then the cells were incubated with $0.1 \%$ naphthol AS-MX phosphate (Sigma, St. Louis, $\mathrm{MO}$ ) and $0.1 \%$ fast blue RR salt (Sigma, St. Louis, MO) in $56 \mathrm{mM}$ 2-amino-2-methyl-1,3-propanediol ( $\mathrm{pH}$ 9.9, Sigma, St. Louis, MO) working solution at room temperature for $10 \mathrm{~min}$, washed with PBS twice and then observed under an optical microscope. More than ten bright field images of ALP staining were taken from each sample and at least 3 parallel samples were stained. As reported by Alm et al. [14], the level of ALP activity was quantified by using an ImageJ program analysis. Briefly, a constant threshold value of the reflection intensity was defined from background and the percentage of ALP positively stained area to total area of the cells was calculated.

\section{Alizarin red S staining}

To evaluate the deposition level of calcium phosphate, the cultured
MSCs were washed with PBS twice, followed by fixation with $4 \%$ paraformaldehyde for $10 \mathrm{~min}$ at room temperature. The fixed cells were incubated with $0.5 \%$ Alizarin red S (Sigma, St. Louis, MO) solution at room temperature for 10 min and extensively washed twice with PBS. The stained cells were observed under an optical microscope. Similarly, Alizarin red $S$ positive areas were quantified by determining the percentage of stained area to total area of the cells using ImageJ.

\section{Oil red $O$ staining}

To evaluate the adipogenic differentiation, Oil red O (Sigma, St. Louis, MO) staining of the cytoplasmic droplets of neutral lipids in the differentiated cells was performed according to the previous research $[15,16]$. The cultured cells were rinsed with PBS and fixed with $4 \%$ paraformaldehyde for $10 \mathrm{~min}$. After treatment with 60\% 2-propanol solution for $5 \mathrm{~min}$, the cells were treated with Oil red O working solution for $5 \mathrm{~min}$ at room temperature. The Oil red $\mathrm{O}$ working solution was prepared by mixing three parts of Oil red O stock solution $(0.3 \mathrm{~g} / \mathrm{mL}$ Oil red O powder in 2-propanol) with two parts of MilliQ water. After that, the stained cells were washed with 60\% 2-propanol solution once and PBS three times to help remove the background. The photomicrographs of the stained cells were captured by optical microscopy. The percentage of cells positively stained by Oil red $\mathrm{O}$ was calculated from the stained area and total area of cells as mentioned above.

\section{Real-time PCR Analysis}

Total RNA was extracted from the cells cultured at different conditions for pre-designated periods by using Sepasol solution a
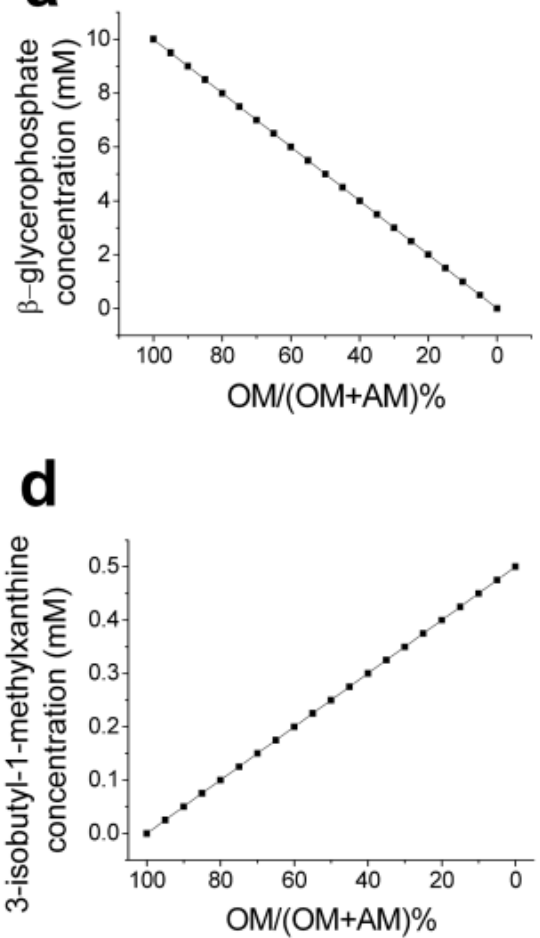

b

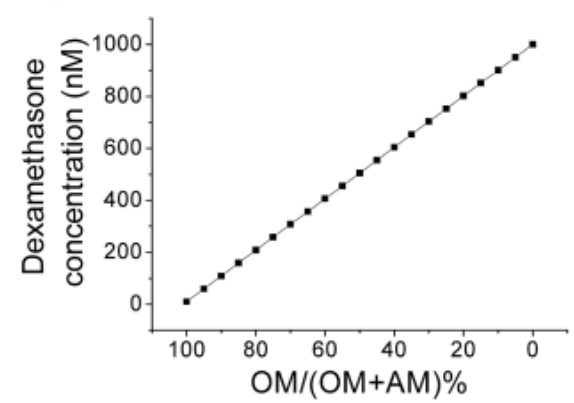

e

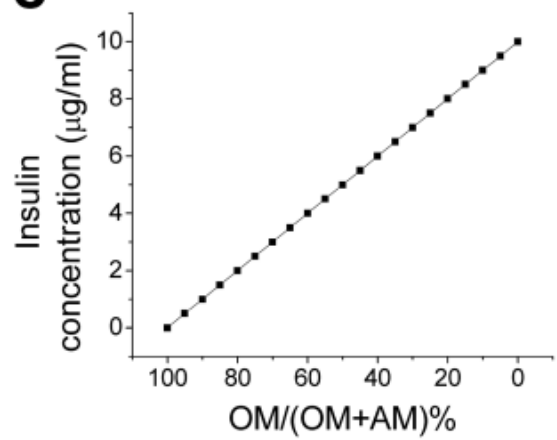

C

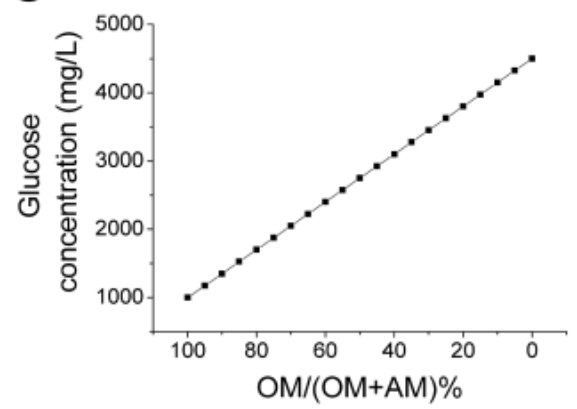

f

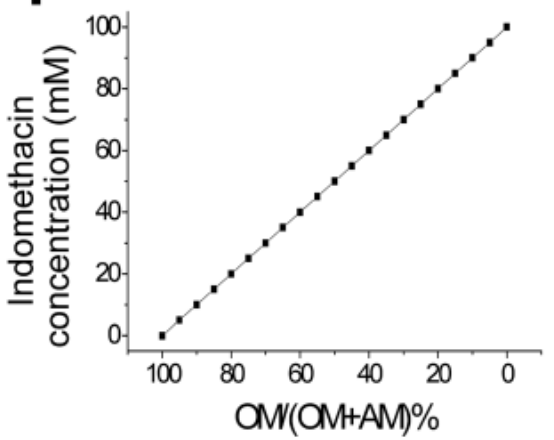

Figure 1: The concentration of osteogenic and adipogenic induction factors. Osteogenic differentiation cocktails contain (a) $\beta$-glycerophosphate ( $\beta$-GP) and (b) dexamethasone (Dex). The concentration of (c) glucose was also changed according to different mixture medium. Adipogenic differentiation cocktails share the same inducing factor, dexamethasone. Besides that, adipognic induction factors used in this study contain (d) 3-isobutyl-1-methylxanthine (IBMX), (e) insulin, and (f) indomethacin (Indo). Among these soluble factors, only the concentration of $\beta$-GP decreased with the increasing percentage of adipogenic medium. 
Citation: Cai R, Nakamoto T, Hoshiba T, Kawazoe N, Chen G (2014) Control of Simultaneous Osteogenic and Adipogenic Differentiation of Mesenchymal Stem Cells. J Stem Cell Res Ther 4: 223. doi:10.4172/2157-7633.1000223

Page 3 of 9

according to the manufacturer's instruction (Nacalai Tesque, Kyoto, Japan). Total RNA $(1.0 \mu \mathrm{g})$ was used as a first strand reaction that included random hexamer primers and murine leukemia virus reverse transcriptase (Applied Biosystems, CA). Real-time PCR was used to quantify $18 \mathrm{~S}$ rRNA, glyceraldehyde-3-phosphate dehydrogenase $(G A P D H)$, Alkaline phosphatase $(A L P)$, secreted phosphoprotein 1 (SPP1, osteopontin), bone sialoprotein 2 (IBSP), SP7 transcription factor (SP7; osterix), peroxisome proliferator-activated receptor gamma $(P P A R G)$, lipoprotein lipase $(L P L)$, fatty acid binding protein 4 (FABP4) and CCAAT/enhancer binding protein (CEBPA), and fatty acid synthase $(F A S N)$. The reaction was performed with $10 \mathrm{ng}$ of cDNA, $90 \mathrm{nM}$ PCR primers, $25 \mathrm{nM}$ PCR probe and FastStart TaqMan Probe Master (Roche Diagnostics Japan, Tokyo, Japan). The expression level of $18 \mathrm{~S}$ rRNA was used as an endogenous control and gene expression level relative to GAPDH was calculated using the comparative $\mathrm{Ct}$ method. The sequences of primers and probes are based on our previous studies as listed in Table $1[15,17]$. The primers and probes were obtained from Applied Biosystems and Hokkaido System Science (Sapporo, Japan).

\section{Statistical analysis}

All data are presented as mean \pm standard deviation (SD), with 3 biological replicates. The statistically significant effects were detected by one-way analysis of variance (ANOVA) with Tukey's post hoc test. All the results were performed in at least three independent experiments. A significant difference is marked as ${ }^{\star}(p<0.05),{ }^{* *}(p<0.01)$, and $* * *(p<0.001)$.

\section{Results}

\section{Osteogenic differentiation of MSCs in mixed osteogenic/ adipogenic medium}

Specific induction factors are necessary to commit MSCs toward osteogenic and adipogenic differentiation. To investigate how the osteogenic induction medium $(\mathrm{OM})$ and adipogenic induction medium (AM) in the mixture medium affect the osteogenic differentiation of MSCs, cells were cultured in mixture medium at various ratios for 3 , 7,14 , and 21 days. ALP was stained acting as the early stage maker of osteogenesis [18-21]. The ALP staining was negative when MSCs were cultured in growth medium after $3,7,14$, and 21 days (Figure 1a), while the cells cultured in mixture medium were positively stained. For the first 3 days of culture, the cells were stained weakly. ALP staining became gradually stronger when the culture time increased. After 21 days, cells cultured in medium at all the mixture ratios were positively stained.

\begin{tabular}{|l|l|l|}
\hline mRNA & & Oligonucleotide \\
\hline 18 S rRNA & & Hs99999901_s1 \\
GAPDH & & Hs99999905_m1 \\
ALP & & 5'-GACCCTTGACCCCCACAAT-3' \\
& & 5'-GCTCGTACTGCATGTCCCCT-3' \\
& & 5'-TGGACTACCTATTGGGTCTCTTCGAGCCA-3' \\
IBSP & Forward & 5'-TGCCTTGAGCCTGCTTCC-3' \\
& Reverse & 5'-GCAAAATTAAGCAGTCTTCATTTTG-3' \\
& Probe & 5'-CTCCAGGACTGCCAGAGGAAGCAATCA-3' \\
SPP1 & Forward & 5'-CTCAGGCCAGTTGCAGCC-3' \\
& Reverse & 5'-CAAAAGCAAATCACTGCAATTCTC -3' \\
& Probe & 5'-AAACGCCGACCAAGGAAACTCACTACC-3' \\
SP7 & Forward & Hs00541729_m1 \\
PPARG & Reverse & Hs01115510_m1 \\
LPL & Probe & Hs00173425_m1 \\
FABP4 & & Hs00609791_m1 \\
FASN & & Hs00188012_m1 \\
CEBPA & & Hs00269972_s1 \\
\hline
\end{tabular}

Table 1: Primers and probes for real-time PCR analysis.
The intensity of ALP staining was also dependent on the ratio of $\mathrm{OM}$ and $\mathrm{AM}$. The cells cultured in mixture medium with an $\mathrm{OM} / \mathrm{AM}$ ratio in the range of 90/10 60/40 were more positively stained than the cells cultured in other mixture medium. The ALP staining was further quantified by image analysis of the percentage of positively stained areas (Figure 1b) [14]. The intensity of ALP staining was almost zero after 3 days of culture and increased with culture time. After being cultured in the mixture medium for 14 and 21 days, the ALP intensity was very high, and the dependence of ALP staining on the composition of mixture medium became remarkably obvious after 14 and 21 days culture. The degree of ALP staining increased when the OM/AM ratio was changed from $100 / 0$ to $90 / 10$, remained maximum at the same level from OM/AM ratio of $90 / 10$ to $60 / 40$, and further decreased below a ratio of $55 / 45$. At mixture ratios of $5 / 95$ and $0 / 100$, the cells were only slightly stained by ALP, even after being cultured for 21 days.

Calcium deposition was examined by Alizarin red S staining, as calcium deposition is a late stage indicator of osteogenic differentiation [20]. MSCs cultured in growth medium showed no calcium deposition. MSCs cultured in the OM/AM mixture medium showed no calcium deposition until the cells were cultured for 21 days (Figure 2a). At day 21, the Alizarin red S staining was strong for the cells cultured in mixture medium with OM/AM ratio of 100/0, 95/5, 90/10, 85/15, $80 / 20,75 / 25,70 / 30$, moderate for cells culture at OM/AM ratio of $65 / 35,60 / 40,55 / 45,25 / 75,20 / 80$, and $15 / 85$, and low for the cells cultured at OM/AM ratio of 50/50, 55/45, 60/40, 65/35, 30/70, 90/10, $95 / 5$, and $0 / 100$. The Alizarin red S staining intensity was quantified by image analysis of the percentage of Alizarin red S stained areas (Figure $2 \mathrm{~b}$ ). The Alizarin red S staining was at the same high level when MSCs were cultured in mixture medium with an OM/AM ratio of 100/0 to $70 / 30$. It showed a decrease from an OM/AM ratio of $65 / 35$ to $30 / 70$ and was much lower when the OM/AM ratio was 55/45, 40/60, 35/65, and 30/70. There was a small peak of Alizarin red S intensity when OM/ AM ratio was in the range of 25/75 15/85. The intensity of Alizarin red $\mathrm{S}$ staining showed a quite low level again when the OM/AM ratio was $10 / 90,5 / 95$ and $0 / 100$.

Based on the ALP and Alizarin red S staining results, a few mixture ratios $(100 / 0,95 / 5,85 / 15,70 / 30,50 / 50,20 / 80$, and 0/100) where MSCs showed distinctly high or low intensity of staining were selected for gene expression experiments. Expression of osteogenic genes ALP, SPP1, $S P 7$, and IBSP were measured after MSCs were cultured for 21 days (Figure 3). Compared with that of cells cultured in growth medium, expression level of these osteogenic genes increased significantly when MSCs were cultured in mixture medium. Expression of the osteogenic genes was shown to be dependent on the composition of the mixture medium. Expression level of $A L P$ by MSCs cultured at OM/AM mixture ratios of $100 / 0,95 / 5,85 / 15$, and $70 / 30$ were higher than that of cells cultured at OM/AM mixture ratios of 50/50, 20/80, and 0/100. Expression level of $S P P 1, S P 7$, and $I B S P$ was high in cells cultured in mixture medium with OM/AM mixture ratio of $100 / 0,95 / 5,85 / 15$, and 70/30. The expression levels were moderate for cells cultured at $\mathrm{OM} / \mathrm{AM}$ mixture ratio of 20/80, and low for cells cultured at OM/AM mixture ratio of 50/50 and $0 / 100 . A L P$ and $S P P 1$ are the early stage genes of osteogenesis, while $S P 7$ and IBSP are the late stage genes. High expression of late stage marker genes was in good agreement with the calcium deposition results. Together with the ALP and Alizarin red $S$ staining, gene expression results indicated that MSCs cultured in mixture medium with $\mathrm{OM}$ percentage greater than $70 \%$ showed strong osteogenic differentiation, and moderate osteogenic differentiation when the OM percentage was in a range of $70 \% \sim 55 \%$. The OM percentage lower than $50 \%$ was not good for osteogenic differentiation, 
Citation: Cai R, Nakamoto T, Hoshiba T, Kawazoe N, Chen G (2014) Control of Simultaneous Osteogenic and Adipogenic Differentiation of Mesenchymal Stem Cells. J Stem Cell Res Ther 4: 223. doi:10.4172/2157-7633.1000223

a

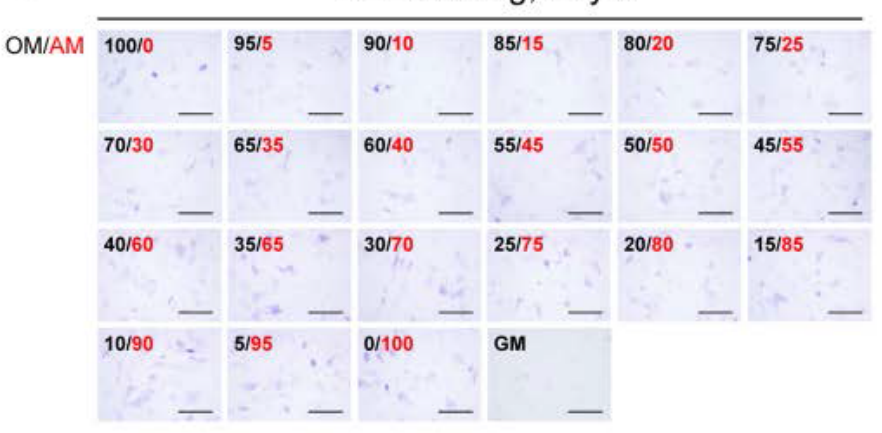

ALP staining, Day 14

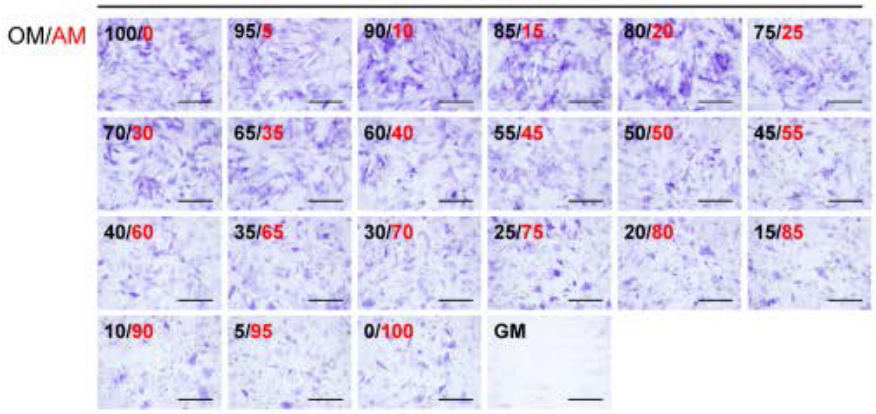

ALP staining, Day 7

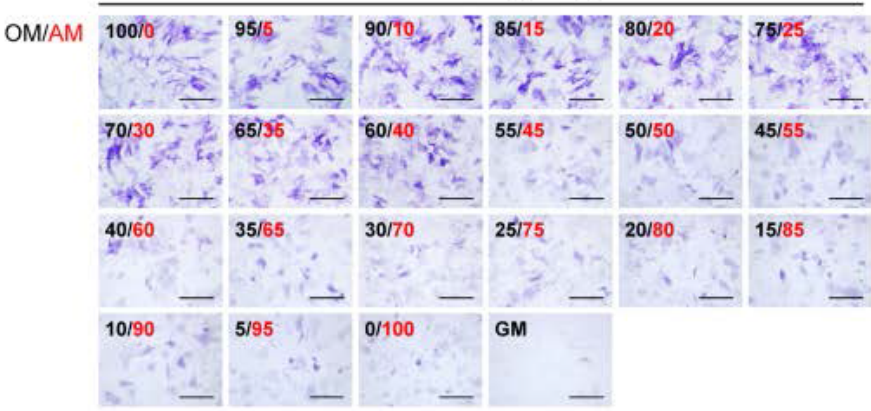

ALP staining, Day 21

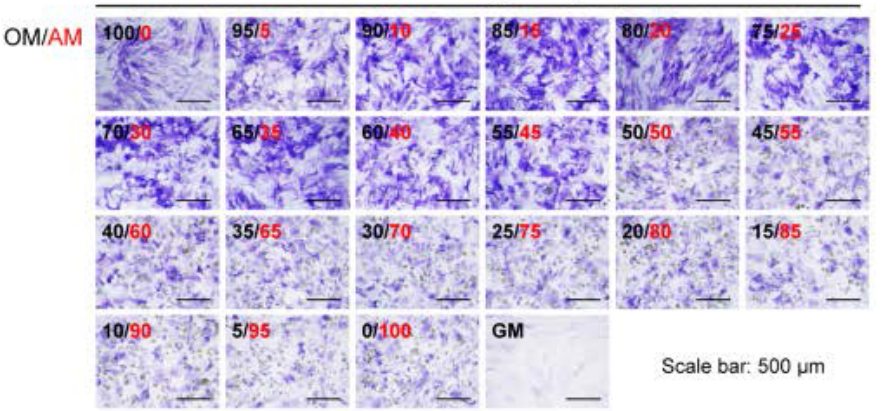

b

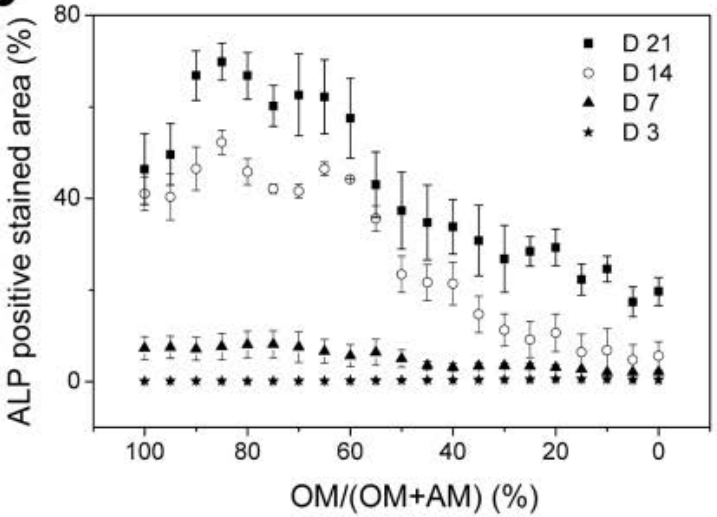

Figure 2: (a) Photomicrographs of ALP staining of MSCs after culture in different ratio of osteogenic medium (OM) and adipogenic medium (AM) for 3, 7, 14, and 21 days. 100/0 indicates the medium containing 100\% OM and $0 \%$ AM, and so forth. GM indicates the growth medium. Scale bar=500 $\mu$ m. (b) Percentage of cells that were positively stained with ALP after culture in different ratio of OM and AM for 21 days. Data represent mean $\pm S D$ ( $n \geq 30, n$ represents the total number of images taken from 3 triplicate wells with more than 10 images from each well).

except the range between $25 \%$ and $15 \%$. During the range between $25 \%$ and $15 \%$, MSCs also showed moderate osteogenic differentiation.

\section{Adipogenic differentiation of MSC in mixed osteogenic/ adipogenic medium}

Adipogenic differentiation of MSCs in the OM/AM mixture medium was investigated by Oil red $\mathrm{O}$ staining and real-time PCR. Oil red $\mathrm{O}$ staining of cells was negative for 3 days culture and slightly positive for 7 days culture, and became strongly positive after 14 and 21 days culture (Figure $4 a$ ). Few cells were positively stained in mixture medium with an OM/AM ratio of 100/0, 95/5, and 90/10. The number of stained cells increased with an increase in AM percentage, and a considerable amount of cells were stained when AM percentage was higher than 50\%. MSCs cultured in growth medium were not positively stained.

Quantification of the positive stained area showed the staining intensity of the cells cultured in mixture medium (Figure $4 \mathrm{~b}$ ). The positive stained area was almost zero when MSCs were cultured in the mixture medium for 3 days and very low after 7 days of culture. The positive stained area showed a significant increase after 14 and 21 days of culture, respectively. The positive stained area was dependent on the composition of the mixture medium. It increased with increasing AM percentage and became plateau when the AM percentage was higher than $75 \%$.

Expression of adipogenesis related genes $L P L, F A B P 4, P P A R G$, 
Citation: Cai R, Nakamoto T, Hoshiba T, Kawazoe N, Chen G (2014) Control of Simultaneous Osteogenic and Adipogenic Differentiation of Mesenchymal Stem Cells. J Stem Cell Res Ther 4: 223. doi:10.4172/2157-7633.1000223

a

\section{OM/AM}

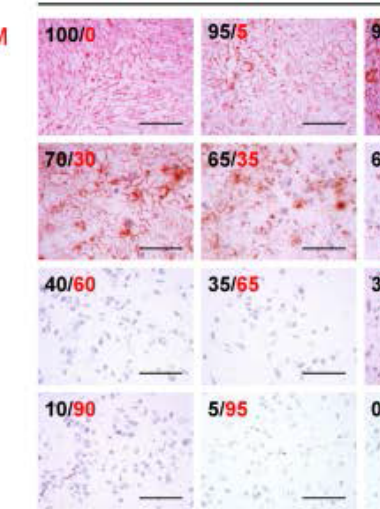

Alizarin red S staining, Day 21

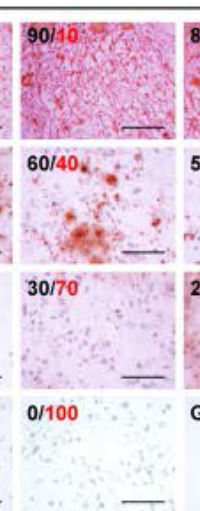

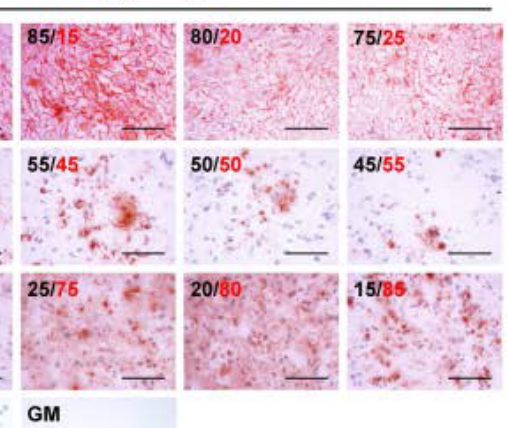

Scale bar: $500 \mu \mathrm{m}$ b

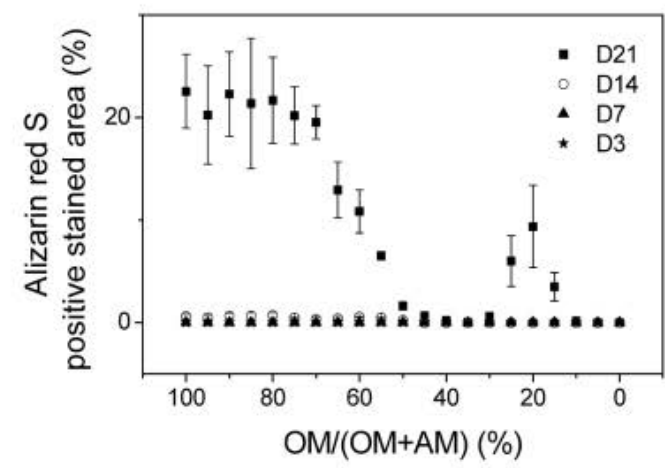

Figure 3: (a) Photomicrographs of Alizarin red S staining of MSCs after culture in different ratio of OM and AM for 3, 7, 14, and 21 days. 100/0 indicates the medium containing $100 \% \mathrm{OM}$ and $0 \% \mathrm{AM}$, and so forth. GM indicates the growth medium. Scale bar=500 $\mu \mathrm{m}$. (b) Percentage of cells that were positively stained with Alizarin red $S$ after culture in different ratio of $O M$ and $A M$ for 21 days. Data represent mean $\pm S D(n \geq 30, n$ represents the total number of images taken from 3 duplicate wells with more than 10 images from each well).

a

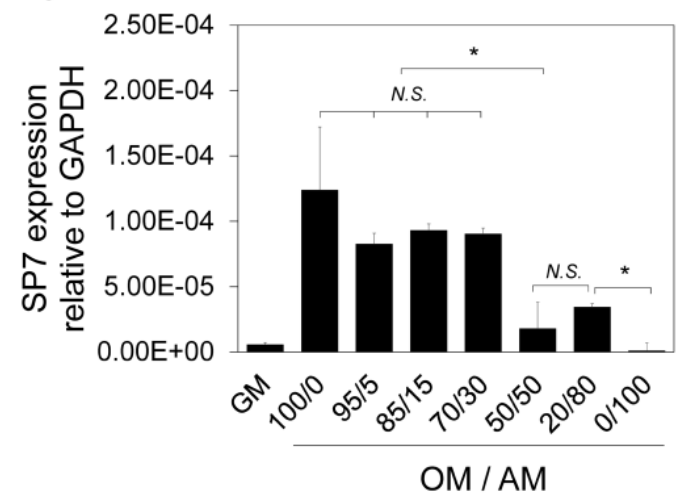

C

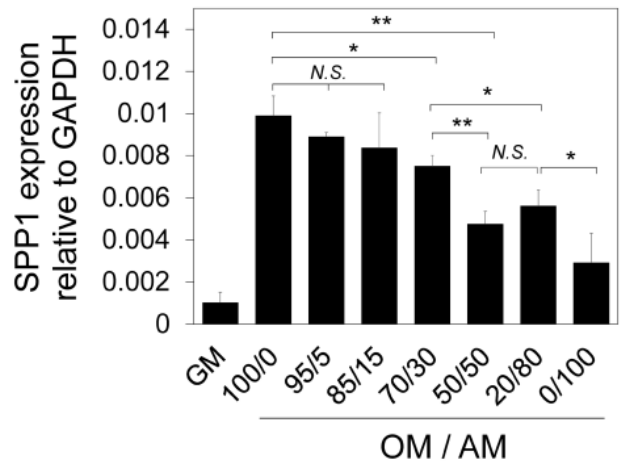

b

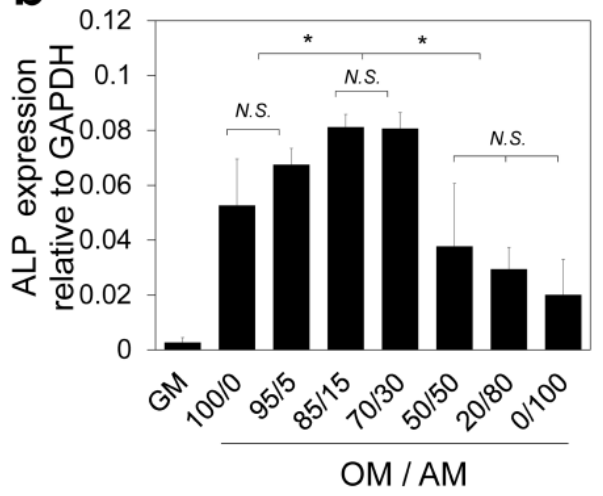

d

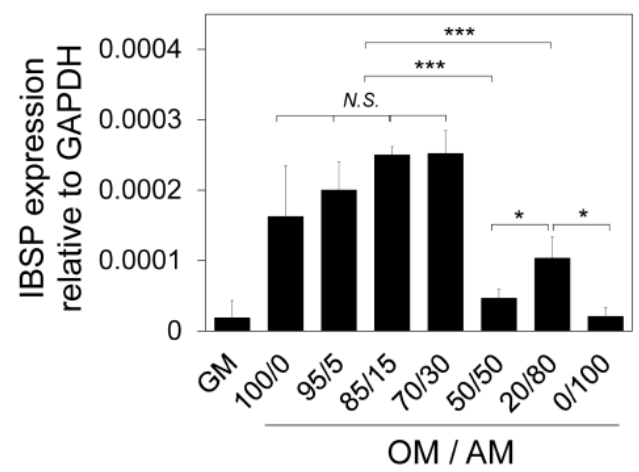

Figure 4: Expression of (a) SP7, (b) $A L P$, (c) IBSP, and (d) SPP1 genes after MSCs were cultured in GM and mixture medium of OM and AM at a ratios of 100/0, $95 / 5,85 / 15,70 / 30,20 / 80$, and $0 / 100$ for 21 days. The gene expression was investigated by real-time PCR analysis. Data represent mean \pm SD $(n=3, n$ represents the number of duplicate samples of cultured cells at the same condition). ${ }^{*}, P<0.05,{ }^{* *}, P<0.01,{ }^{* * *}, P<0.001$.

CEPBA, and FASN, was quantified by real-time PCR (Figure 5). Expression of the genes was very low when MSCs were cultured in growth medium. All the genes showed low expression in mixture medium containing less AM, and higher expression in mixture medium containing more AM. The expression of the genes increased with the AM percentage. For $P P A R G$ and $L P L$, expression levels were high when
AM percentage was greater than $70 \%$. For CEBPA, FABP4, and FASN, their expression reached the highest level when AM percentage was greater than $80 \%$.

\section{Discussion}

Osteogenic and adipogenic differentiation are the most important 
a

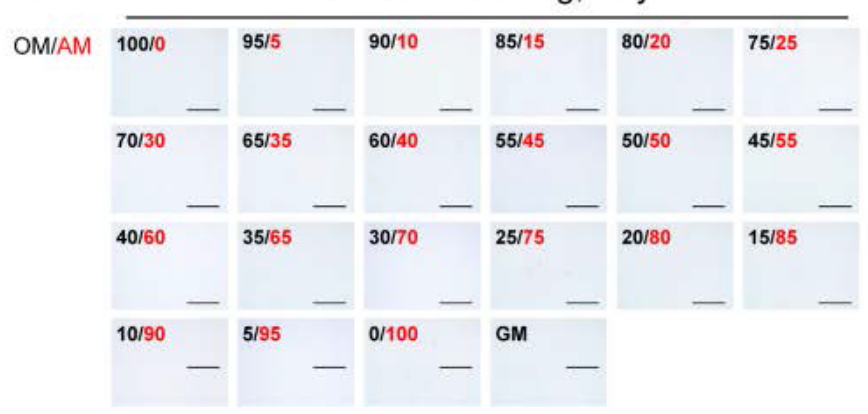

Oil red O staining, Day 14

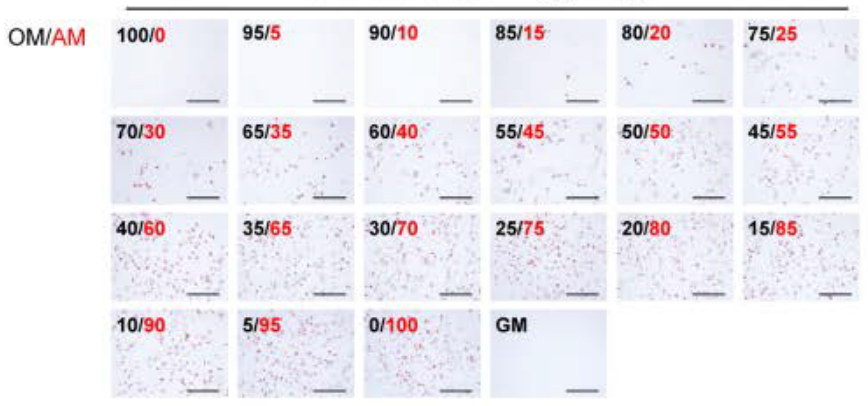

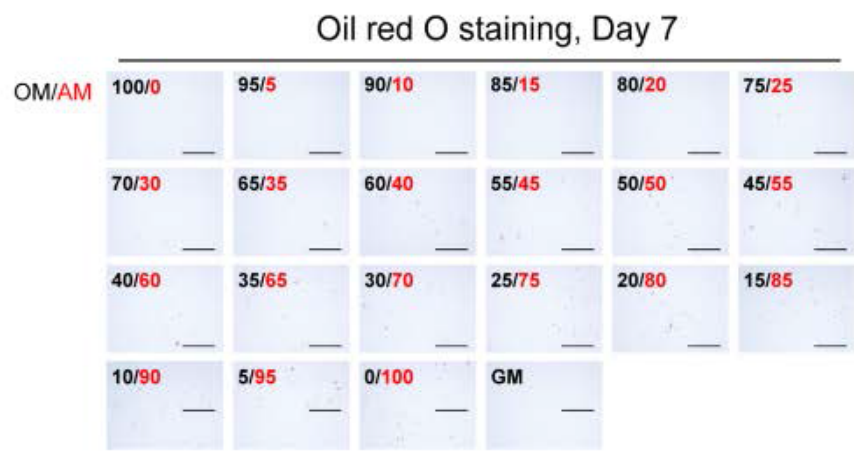

Oil red O staining, Day 21

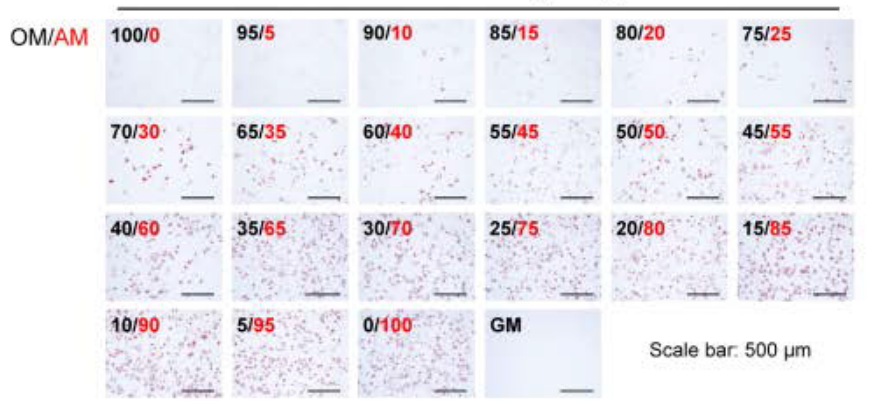

b

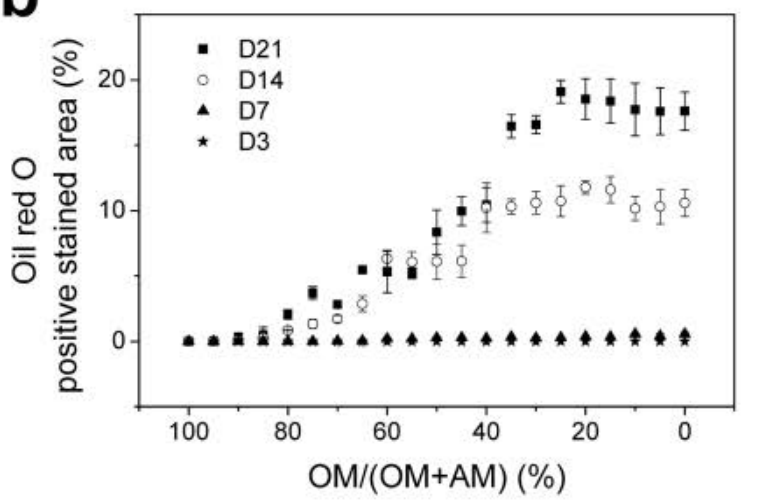

Figure 5: (a) Photomicrographs of Oil red O staining of MSCs after culture in different ratio of OM and AM for $3,7,14$, and 21 days. 100/0 indicates the medium containing $100 \% \mathrm{OM}$ and $0 \% \mathrm{AM}$, and so forth. GM indicates the growth medium. Scale bar=500 $\mu \mathrm{m}$. (b) Percentage of cells that were positively stained with Oil red $O$ after culture in different ratio of $O M$ and $A M$ for 21 days. Data represent mean $\pm S D(n \geq 30, n$ represents the total number of images taken from 3 duplicate wells with more than 10 images from each well).

characteristics of MSCs. Control of their osteogenesis and adipogenesis is very important for tissue engineering and regenerative medicine $[12,22,23]$. Usually, differentiation of MSCs is studied by culturing them in a specific differentiation medium. The classical cocktail of induction factors for osteogenic and adipogenic differentiation of MSCs in vitro has been well established. The composition of medium has significant effect on the differentiation of MSCs. The osteogenic induction medium and adipogenic induction medium have some similar components.

However, it is unclear whether MSCs can simultaneously differentiate to osteogenic and adipogenic lineages and whether the medium components can balance the osteogenesis and adipogenesis of MSCs. In this study, the effect of these two types of induction media in mixture on balance of the osteogenic and adipogenic differentiation of MSCs was investigated. Simultaneous osteogenic and adipogenic differentiation of MSCs was induced by culturing MSCs in the mixture medium with different ratio of OM and AM. After they were cultured in the mixture medium for 21 days, MSCs showed strong osteogenic differentiation in the mixture medium containing an OM/AM ratio from 100/0 70/30. Moderate osteogenic differentiation was observed at $\mathrm{OM} / \mathrm{AM}$ ratio of $70 / 30 \sim 50 / 50$ and $25 / 75 \sim 15 / 85$. OM/AM ratios of 50/50 25/75 and 15/85 0/100 showed low levels of osteogenic differentiation. Adipogenic differentiation of MSCs in the mixture medium showed a simple dependence on the AM percentage. High ratio of $\mathrm{AM}$ resulted in strong adipogenic differentiation, while at low ratio adipogenic differentiation was weak.

Therefore, the mixture medium can be divided into 5 ranges to balance the osteogenic and adipogenic differentiation of MSCs. They are OM/AM ratios of 100/0 70/30 for strong osteogenic differentiation and weak adipogenic differentiation, 70/30 50/50 for moderate 


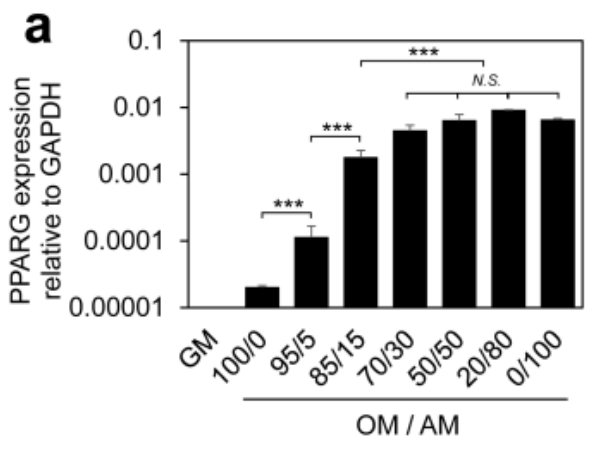

C

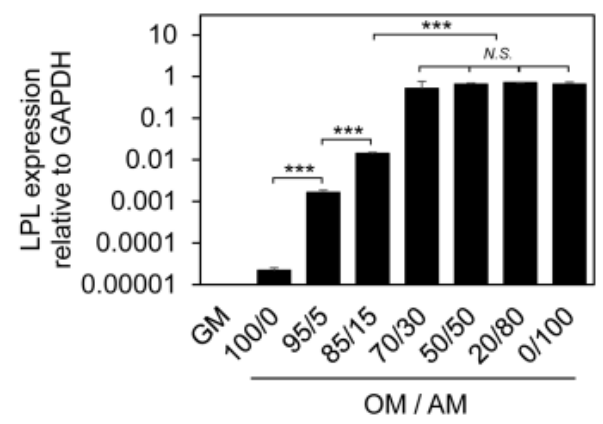

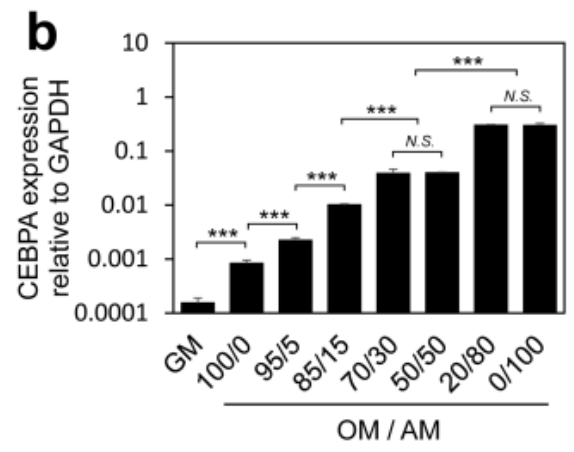

Figure 6: Expression of (a) PPARG (b) CEBPA (c) $L P L$ (d) FABP4 and (e) FASN genes after MSCs were cultured in GM and mixture medium of OM and AM at a ratios of $100 / 0,95 / 5,85 / 15,70 / 30,20 / 80$, and $0 / 100$ for 21 days. The gene expression was investigated by real-time $P C R$ analysis. Data represent mean \pm SD ( $n=3$, $\mathrm{n}$ represents the number of duplicate samples of cultured cells at the same condition). ${ }^{*}, P<0.05,{ }^{* *}, P<0.01,{ }^{* * *}, P<0.001$.

osteogenic differentiation and moderate adipogenic differentiation, $50 / 50 \sim 25 / 75$ for weak osteogenic differentiation and moderate adipogenic differentiation, 25/75 15/85 for moderate osteogenic differentiation and strong adipogenic differentiation, 15/85 0/100 for weak osteogenic differentiation and strong adipogenic differentiation.

A large number of evidence suggests that an inverse correlation exists between osteogenesis and adipogenesis [11,24,25], mainly based on different culture supplements. The up-regulation of osteogenic differentiation is associated with down-regulation of adipogenic differentiation, and vice versa [10]. The general trend of osteogenic and adipogenic differentiation in the mixture medium was consistent with the previously reported results. However, the results in this study showed more detailed information on the balance of simultaneous osteogenic and adipogenic differentiation by the mixture medium.

Typically, the culture supplements regulate key transcription factors that control the balance of osteogenesis and adipogenesis. Dex and $\beta$-GP are the two inductors for osteogenic differentiation of MSCs [26]. Dex, IBMX, insulin, and Indo are the main inductors for adipogenic differentiation. The concentration of these inductors in the OM/AM mixture medium is shown in Figure 6. The favorable osteogenic differentiation at high percentages of osteogenic induction medium and favorable adipogenic differentiation at high percentages of adipogenic induction medium should be due to the appropriate concentration range of their respective induction factors.

Dex, a synthetic glucocorticoid, has been reported to be an essential soluble factor for osteogenesis of MSCs, but it has also been recognized as a adipogenesis promoter at a higher concentration [9]. Many reports found that Dex modulates osteogenesis and adipogenesis in MSCs by regulating SP7 expression [25], Wnt signaling [27], TAZ [28], and SPP1 [29] expression. The Dex concentration in the mixture medium with an
$\mathrm{OM} / \mathrm{AM}$ ratio of 100/0 70/30 was 10 300 nM. Alm et al. [14] reported that treatment with $10 \mathrm{nM}$ Dex was not as effective as with $100 \mathrm{nM}$ Dex.

For mineralization, the late stage of osteogenesis, the presence of both calcium and phosphate ions is necessary. $\beta$-GP serves as a crucial source of calcium phosphate. Furthermore, the calcium phosphate deposition is highly dose dependent on the concentration of $\beta$-GP. The $\beta$-GP concentration decreased with the change of OM/AM ratios from $100 / 0$ to $0 / 100$. The increase of Dex concentration and decrease of $\beta$-GP concentration in the mixture medium explains the best osteogenesis-stimulation effect of mixture medium with an OM/AM ratio of $100 \sim 70 / 30$. The reason for the stimulation effect at an OM/AM ratio of $25 / 75 \sim 15 / 85$ is not clear. It probably is due to the synergistic effects from all the combined components in the mixture medium.

During the adipogenesis of MSCs, high concentration of Dex usually induces expression of $C E B P A$ and $P P A R G$, which act as two key transcription factors of adipogenic differentiation [30]. The positive effect of Dex on adipogenic differentiation of MSCs can be amplified by cAMP-elevating agents such as IBMX [31]. On other hand, IBMX can up-regulate the expression of $P P A R G$ and $L P L$ and also down-regulate osteogenic gene makers such as RUNX2 and SPP1 by activation of protein kinase A. Therefore, Dex and IBMX play important roles in modulating the balance between osteogenesis and adipogenesis. Beside these two adipogenic factors, insulin and indomethacin can also promote adipogenic differentiation by stimulating $P P A R G$ or binding with PPARG [32].

We also analyzed the main transcription factor for osteogenic differentiation of MSCs, SP7. SP7 can induce preosteoblasts to differentiate into mature osteoblasts that mineralize and express high level of osteogenic-specific genes, such as bone sialoprotein (IBSP). 
Citation: Cai R, Nakamoto T, Hoshiba T, Kawazoe N, Chen G (2014) Control of Simultaneous Osteogenic and Adipogenic Differentiation of Mesenchymal Stem Cells. J Stem Cell Res Ther 4: 223. doi:10.4172/2157-7633.1000223

Frequently, LPL expression and observation of oil droplets are used as the early and late markers of adipogenesis, respectively. During the terminal differentiation of adipogenesis, adipocytes markedly increase the expression of proteins related to lipid metabolism, such as FABP4, an adipocyte-specific fatty acid protein, also identified as $a P 2$. All the adipogenesis related genes were up-regulated with an increase in the AM percentage. This should be attributed to the increase of the adipogenesis induction factors.

These results established an important relationship between osteogenesis and adipogenesis. In bone marrow, osteoblasts and adipocytes are derived from the mesenchymal stem cells, transcription factors and genes involved in these two lineages could be manipulated by induction factors to favor osteogenic or adipogenic differentiation of MSCs. It has been reported that PPARG is proposed as a major factor in lineage skewing, promoting adipogenesis at the expense of osteogenesis [13]. PPARG activating led to ROS accumulation cause apoptosis of osteoblasts [12], and PPARG silencing stimulated a higher level of matrix mineralization [24]. Our results indicate that when the normal osteogenesis of MSCs is disrupted under interventions, adipogenesis dominates the differentiation process, and the reverse is also true. Shifting in balance between these two processes is regulated by involved transcription factors, which can be activated or silenced by soluble factors.

\section{Conclusion}

In conclusion, the simultaneous osteogenic and adipogenic differentiation of MSCs was studied by culturing MSCs in mixture medium. The composition of the mixture medium showed a significant effect on the osteogenesis and adipogenesis of MSCs. Adipogenic differentiation became stronger with the increase of adipogenic differentiation medium percentage and decrease of osteogenic differentiation medium percentage in the mixture medium. Osteogenic differentiation happened at two different ranges of compositions. It was very strong when the percentage of osteogenic differentiation medium was high and decreased to very weak levels when the percentage decreased to $50 \%$. However, osteogenic differentiation became strong again when the percentage of osteogenic differentiation medium was in the range of $25 \%$ to $15 \%$. Balancing of simultaneous osteogenic and adipogenic differentiation of MSCs by the mixture medium should provide useful information for stem cell studies and tissue regeneration.

\section{Acknowledgment}

This work was supported by the World Premier International Research Center Initiative on Materials Nanoarchitectonics and a Grant-in-Aid for Scientific Research (B) from the Ministry of Education, Culture, Sports, Science and Technology of Japan.

\section{References}

1. Pittenger MF, Mackay AM, Beck SC, Jaiswal RK, Douglas R, et al. (1999) Multilineage potential of adult human mesenchymal stem cells. Science 284 143-147. [PubMed]

2. Robinson SN, Ng J, Niu T, Yang H, McMannis JD, et al. (2006) Superior ex vivo cord blood expansion following co-culture with bone marrow-derived mesenchymal stem cells. Bone Marrow Transplant 37: 359-366. [PubMed]

3. Pei M, He F, Kish VL (2011) Expansion on Extracellular Matrix Deposited by Human Bone Marrow Stromal Cells Facilitates Stem Cell Proliferation and Tissue-Specific Lineage Potential. Tissue Engineering Part A 17: 3067-3076.

4. Andrade PZ, dos Santos F, Almeida-Porada G, da Silva CL, JM SC (2010) Systematic delineation of optimal cytokine concentrations to expand hematopoietic stem/progenitor cells in co-culture with mesenchymal stem cells. Mol Biosyst 6: 1207-1215. [PubMed]

5. Pattappa G, Thorpe SD, Jegard NC, Heywood H, de Bruijn JD, et al. (2012)
Continuous and uninterrupted oxygen tension influences the colony formation and oxidative metabolism of human mesenchymal stem cells. Tissue Eng Part C Methods.

6. Basciano L, Nemos C, Foliguet B, de Isla N, de Carvalho M, et al. (2011) Long term culture of mesenchymal stem cells in hypoxia promotes a genetic program maintaining their undifferentiated and multipotent status. BMC Cell Biol 12: 12 [PubMed]

7. Mauney JR, Volloch V, Kaplan DL (2005) Matrix-mediated retention of adipogenic differentiation potential by human adult bone marrow-derived mesenchymal stem cells during ex vivo expansion. Biomaterials 26: 61676175.

8. Discher DE, Mooney DJ, Zandstra PW (2009) Growth Factors, Matrices, and Forces Combine and Control Stem Cells. Science 324: 1673-1677. [PubMed]

9. Vater C, Kasten P, Stiehler M (2011) Culture media for the differentiation of mesenchymal stromal cells. Acta Biomaterialia 7: 463-477. [PubMed]

10. Beresford JN, Bennett JH, Devlin C, Leboy PS, Owen ME (1992) Evidence for an Inverse Relationship between the Differentiation of Adipocytic and Osteogenic Cells in Rat Marrow Stromal Cell-Cultures. Journal of Cell Science 102: $341-351$.

11. Liu HY, Wu ATH, Tsai CY, Chou KR, Zeng R, et al. (2011) The balance between adipogenesis and osteogenesis in bone regeneration by platelet-rich plasma for age-related osteoporosis. Biomaterials 32: 6773-6780. [PubMed]

12. Botolin S, McCabe LR (2007) Bone loss and increased bone adiposity in spontaneous and pharmacologically induced diabetic mice. Endocrinology 148 198-205. [PubMed]

13. Bruedigam C, Eijken M, Koedam M, van de Peppel J, Drabek K, et al. (2010) A New Concept Underlying Stem Cell Lineage Skewing That Explains the Detrimental Effects of Thiazolidinediones on Bone. Stem Cells 28: 916-927.

14. Alm JJ, Heino TJ, Hentunen TA, Väänänen HK, Aro HT (2012) Transient 100 nM Dexamethasone Treatment Reduces Inter- and Intraindividual Variations in Osteoblastic Differentiation of Bone Marrow-Derived Human Mesenchymal Stem Cells. Tissue Engineering Part C: Methods: 448.

15. Hoshiba T, Kawazoe N, Tateishi T, Chen G (2010) Development of Extracellula Matrices Mimicking Stepwise Adipogenesis of Mesenchymal Stem Cells. Advanced Materials 22: 3042-3047.

16. Aldridge A, Kouroupis D, Churchman S, English A, Ingham E, et al. (2013) Assay validation for the assessment of adipogenesis of multipotential stromal cells--a direct comparison of four different methods. Cytotherapy 15: 89-101. [PubMed]

17. Hoshiba T, Kawazoe N, Tateishi T, Chen G (2009) Development of stepwise osteogenesis-mimicking matrices for the regulation of mesenchymal stem cell functions. J Biol Chem 284: 31164-31173. [PubMed]

18. Siffert RS (1951) The Role of Alkaline Phosphatase in Osteogenesis. Journal of Experimental Medicine 93: 415-448.

19. Caverzasio J, Manen D (2007) Essential role of wnt3a-mediated activation of mitogen-activated protein kinase p38 for the stimulation of alkaline phosphatase activity and matrix mineralization in C3H10T1/2 mesenchymal cells. Endocrinology 148: 5323-5330.

20. Nakashima K, de Crombrugghe B (2003) Transcriptional mechanisms in osteoblast differentiation and bone formation. Trends in Genetics 19: 458-466.

21. Corn DJ, Kim YH, Krebs MD, Mounts T, Molter J, et al. (2013) Imaging early stage osteogenic differentiation of mesenchymal stem cells. Journal of Orthopaedic Research 31: 871-879.

22. Hoshiba T, Kawazoe N, Chen G (2012) The balance of osteogenic and adipogenic differentiation in human mesenchymal stem cells by matrices that mimic stepwise tissue development. Biomaterials 33: 2025-2031. [PubMed]

23. Chen YH, Yeh FL, Yeh SP, Ma HT, Hung SC, et al. (2011) Myocyte Enhancer Factor-2 Interacting Transcriptional Repressor (MITR) Is a Switch That Promotes Osteogenesis and Inhibits Adipogenesis of Mesenchymal Stem Cells by Inactivating Peroxisome Proliferator-activated Receptor $\mathrm{y}-2$. Journal of Biological Chemistry 286: 10671-10680.

24. Lee MJ, Chen HT, Ho ML, Chen CH, Chuang SC, et al. (2013) PPAR gamma silencing enhances osteogenic differentiation of human adipose-derived mesenchymal stem cells. Journal of Cellular and Molecular Medicine 17: 11881193. 
Citation: Cai R, Nakamoto T, Hoshiba T, Kawazoe N, Chen G (2014) Control of Simultaneous Osteogenic and Adipogenic Differentiation of Mesenchymal Stem Cells. J Stem Cell Res Ther 4: 223. doi:10.4172/2157-7633.1000223

25. Mikami Y, Lee M, Irie S, Honda MJ (2011) Dexamethasone modulates osteogenesis and adipogenesis with regulation of osterix expression in rat calvaria-derived cells. Journal of Cellular Physiology 226: 739-748.

26. Zhang Y, Khan D, Delling J, Tobiasch E (2012) Mechanisms Underlying the Osteo- and Adipo-Differentiation of Human Mesenchymal Stem Cells. The Scientific World Journal 2012: 1-14. [PubMed]

27. Taipaleenmäki $H$, Abdallah BM, AIDahmash $A$, Säämänen $A M$, Kassem $M$ (2011) Wnt signalling mediates the cross-talk between bone marrow derived pre-adipocytic and pre-osteoblastic cell populations. Experimental Cell Research 317: 745-756. [PubMed]

28. Hong JH, Hwang ES, McManus MT, Amsterdam A, Tian Y, et al. (2005) TAZ a Transcriptional Modulator of Mesenchymal Stem Cell Differentiation. Science 309: 1074-1078. [PubMed]
29. Chen Q, Shou P, Zhang L, Xu C, Zheng C, et al. (2014) An Osteopontin-Integrin Interaction Plays a Critical Role in Directing Adipogenesis and Osteogenesis by Mesenchymal Stem Cells. Stem Cells 32: 327-337.

30. Cristancho AG, Lazar MA (2011) Forming functional fat: a growing understanding of adipocyte differentiation. Nat Rev Mol Cell Biol 12: 722-734. [PubMed]

31. Kim E-K, Lim S, Park J-M, Seo JK, Kim JH, et al. (2012) Human mesenchyma stem cell differentiation to the osteogenic or adipogenic lineage is regulated by AMP-activated protein kinase. Journal of Cellular Physiology 227: 1680-1687.

32. Smith PJ, Wise LS, Berkowitz R, Wan C, Rubin CS (1988) Insulin-like growth factor-I is an essential regulator of the differentiation of 3T3-LI adipocyte. The Journal of biological chemistry 263: 9402-9408. 